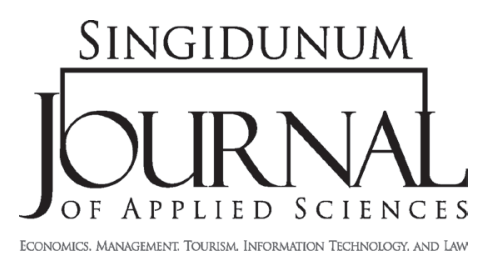

\section{HOW TO WRITE RESEARCH ARTICLES IN COMPUTING AND ENGINEERING DISCIPLINES}

\author{
Ivan Stojmenović, ${ }^{1,}$, Veljko Milutinović ${ }^{2}$ \\ ${ }^{1}$ University of Belgrade, Department of Computer Engineering, \\ 73 Bulevar Kralja Aleksandra Street, Belgrade, Serbia \\ ${ }^{2}$ University of Ottawa, SITE, Canada
}

\begin{abstract}
:
This article advocates a general way of presenting research articles on any topic and in any field related to computer science, information technology, and engineering disciplines. The key advice to a successful presentation is to repeat the description of main contribution four times: in the title, abstract, introduction (or chapter 1) and in the text. That is, to make readable, appealing, and as complete as possible versions of the work using the order of 10,100, 1000 and 10,000 words. This corresponds to the decreasing portion of readers for corresponding parts of the article. To the extent possible, each of these parts should address, in this order: the problem statement, existing solutions, the new solution(s), assumptions and limitations, analysis, simulation and comparison with best competing solutions.
\end{abstract}

SINGIDUNUM JOURNAL 2012, 9 (1): 42-50

ISSN 2217-8090

UDK 001.81:[007:004]

Original paper/Originalni naučni rad

\section{Key words:}

computer science, original paper, abstract, title.

\section{MOTIVATION FOR WRITING AND READING THIS ARTICLE}

The major goal of this paper is to serve as a guideline for the organization of research presentations in written form. The major purpose of the entire effort is to make research presentations as easy to comprehend as absolutely possible. "The process of clarifying your thinking, of which writing papers is one aspect, is a valuable part of improving your research" (Ernst, 2005). The same structure is valid for thesis work, as well as for conference and journal publications, or technical reports for research sponsors.
The motivation to write this article came from the observation that the vast majority of papers in journals, conference proceedings, and the majority of observed oral presentations do not follow the basic structure, as outlined in the abstract here. In most cases, research results are obscured by poor presentation. It is not possible to quickly understand, either the essence of the presented contribution, or the most important research details. This is happening despite the presentation advice appearing to be quite natural and effective for easy understanding of contributions made in a given article. "If you do not write well, why should readers believe you were any more careful in the research itself?" (Ernst, 2005). 
Writing contributions in a form that other people can understand is a very slow process. Excellent presentation also brings some risks for the evaluation, due to "noise in the refereeing system" (Ernst, 2005). A novel idea, well presented, can be claimed to be too simple or even trivial by reviewers, and the judgment is subjective. This author believes in simplicity of any original idea as its ultimate advantage. A number of papers describe new ideas only via programming codes, or complicated diagrams, without presenting clear concise descriptions and/ or figures and illustrations on how they work. This style could potentially bring favorable opinions from reviewers with similar attitude on presentation, or without real desire to understand the details. A wellunderstood idea also brings the risk of being identified as already existing by reviewers. When it is novel and if well understood, it also becomes easier to identify the drawbacks and criticize it. If a problem statement has a new name, different from the name well-known in the community (meaning that the literature review is not done properly), it becomes difficult to identify proper referees for the article and/ or to properly judge its contribution. In summary, there are also reasons not to apply some pieces of advice from this article, if the publication of an article is the sole motivation.

This article places particular attention to four key parts of a research report: the title, abstract, introduction, and to the main body of text. Each of them should be self-contained and complete to the greatest possible extent, because there are four different types of readers. Among those who will ever see any part of a particular work, perhaps an estimated $80 \%$ (the numbers are subjective, based on experience of authors) will see only the title, $15 \%$ will read the abstract (and possibly parts of the conclusion in search for information missing in the abstract), $4 \%$ will also read the introduction, and the remaining $1 \%$ will meaningfully go through the whole paper (some of them may fully read and analyze it, depending on their particular interests and skills, content value, and its presentation).

"A paper should communicate the main ideas of your research early and clearly" (Ernst, 2005). Clear and appealing text in one part increases the chances that a reader will go to the next part and eventually use and cite the work. The readers could be important persons: thesis examiners, reviewers of conferences and journal submissions. They all have limited time to spend on a particular article and time should be used wisely, to make the greatest possible reading and understanding progress for a given amount of time. Reading time normally grows with the clarity and usefulness of text and presentation. Misleading title narrows the readership. If the abstract does not clearly state the contribution made, and fails to attract the reader, then the reader may not advance to the introduction (or the first chapter of a thesis). Sometimes it is beneficial to convince an important reader (examiner, reviewer) that there is no need to study much the main body of the research report, if the introductory text properly presented the contribution and gave a clear picture of the coming text. "A reader who understands the structure and big ideas can better appreciate the details" (Ernst, 2005).

\section{RESEARCH AND PROBLEM SOLVING}

Is research a problem solving exercise? Are there some research activities that do not solve any problems? We believe that this is not the case. If there is no problem to be solved than the related activity could be a development, implementation, or another type of work. Consequently, any research article should make a clear problem statement.

We were frequently faced with articles, even grant proposals, that in fact do not have any real problem to be solved. An example is description of a "novel" software architecture whose evaluation remains fully subjective, without defining any problem to solve. This does not mean that proposing a new architecture is not a valid research problem. However, it so only when the integral parts of doing research are addressed in the article: what problem is resolved by the design of a new architecture, what existing architectures are, what are assumptions and limitations made, how new architecture compares with existing ones etc.

Another example is research article (Stojmenović, 2000) on teaching recursion in the first computer science course. Existing textbooks suggested complicated or no proofs that recursive Fibonacci numbers and binomial coefficients algorithms have exponential time complexities (existing teaching solutions), which were proven on final exams to not be well understood by students. Article (Stojmenović, 2000) described an elegant proof in two lines, easily understood by all students. The proof does not have to be original itself; the main novelty is in applying this proof in the classroom. In general, the identification and application of existing concepts and techniques to new domains and problem statements is a research activity and represents contributions. 


\section{SELECTION OF 'TITLE'}

The selected title of an article should enable the expert to figure out the essence of the basic idea(s) and the main contribution(s), even without reading the paper (Milutinović, 1996). Further, the title should induce the reader to think deeply over the philosophy of the contribution described in the paper (Milutinović, 1996). An example is the title of Kalosha et al. (2008). It states the problem (georouting), the area (sensor networks), desired properties (beaconless, guaranteed delivery) and essence of new idea (select and protest based). Proper title leads to more hits in Google Scholar searches. An imprecise title may bring wrong referees or examiners for the work, and less appreciation.

\section{ABSTRACT: PROTECTING THE CONTRIBUTION}

In our opinion, the abstract is the most important part of a research article. Suppose that one has five minutes of time for an oral presentation, or a few hundred words of written space to protect a new idea and contribution and gain deserved recognition. Note that in many cases abstracts are offered freely while the whole paper needs time (and sometimes also financial resources) to download or acquire. Examples are CiteSeer and Springer databases. How would this time or space be used wisely?

The time/space limitation is almost naturally enforced. Researchers may not pay attention to an article if the abstract does not make convincing statements. Instead of listing topics covered in the article, abstract should convey the essential information found in the paper. The author should make an effort to claim the contribution properly at the most visible place, and not expect readers to do so. Consequently, a good idea may not be noticed by the research community, and those who reinvent it at a later time will get credit instead of the initial inventor (Milutinović, 1996).

The authors generally agree with the five part structure of the abstract as described by Milutinović (1996), as follows (using a simple and concise language):

a) Problem statement of the research under consideration;

b) A short list of existing solutions and what their drawbacks are, from the point of view of the above defined problem statement; c) Essence of the proposed solution, and why it is expected to be better under the same conditions;

d) What type of analysis was done to show that the proposed solution is really better than any of the existing ones, from both the performance and the complexity points of view;

e) What the major numerical highlights of the analysis are. Here we recommend also some qualitative (non-numeric) highlights that may be more feasible and helpful in many cases.

The length of each part should be flexible. For example, the proposed solution could be the first one (a) simple statement with few words for (b), while the new idea (c) may take about half of the abstract to express with sufficient intuition.

The abstract should be written for researchers that are familiar with the research area, and can grasp the contribution easily. Some of them could have worked on the same or related problems. A clear abstract is the key to having the work properly credited in other people's work. One should envision literature reviews of forthcoming papers by other researchers. They could simply "cut and paste" the abstract into their articles, which then serves as an extended patent protection. This could be even done semiautomatically, as others do not really need to read or understand the whole article, to cite it properly. If an abstract is without proper content, citations to the article in other papers may not be very informative either. Should they be? It is the author's obligation in the first place to properly describe the idea with limited space, without expecting that someone else will do this later on behalf of the authors.

Examiners and reviewers will especially appreciate such an abstract to have a friendly start with the text, and obtain a clear picture in a very short period of time. Misleading abstracts are unfortunately quite common practice in research literature. The limited space is too often simply wasted by writing general sentences about the field and excessive explanations about the problem, that should be part of the introduction or Chapter 1. It is best to first answer the above five questions, then see whether there is space left to say anything else.

This structure is also suitable for performance evaluation type of articles. In a performance evaluation based article, the problem is to determine the best protocol under various conditions. Existing performance evaluations are existing solutions. What are their drawbacks? Why is this evaluation 
novel, and what new insights about the protocols are gathered? How does performance evaluation data in this article compare to previous ones?

Survey type of articles however may have a different presentation style. A survey should describe all relevant solutions, classify them according to assumptions made and some properties (that is, present a taxonomy), and draw some conclusions. The contribution of a survey article should still be clarified. It could be the first survey on a given topic or problem, or may present sufficient novel material not covered in existing tutorials, or it could give a taxonomy not seen previously. The survey may also properly describe existing solutions, in a clear and concise manner, compared to existing surveys. A statement of gain the reader will obtain by reading the survey or tutorial article is needed in the abstract. Detailed instructions for writing survey articles can be found on the website of the second author.

\section{CONTENT OF INTRODUCTION OR CHAPTER 1}

In brief, the introduction of a paper intended to be published in a journal or conference, or Chapter 1 of a master or doctoral thesis, should present the same content (summary of the article), in the same order, as the abstract, with more space provided. Normally it is about ten times longer as a rough approximation. There is also space to address some possibly additional items, such as motivation.

While an introduction is normally a single section, Chapter 1 should have separate headings to address the structure and natural flow in the explanation. The abstract may be sufficient for an expert in the field or researchers who have worked on the same problem. The Introduction or Chapter 1 should suffice to comprehend the essence of contribution for people generally working in the area. They should be able to correctly understand what the important aspects of the contribution are, and how good the contribution is.

In our view, the introduction or Chapter 1 should present sufficient information, and be sufficiently self-contained, so that important readers and evaluators do not need to read the rest of text, being assured in the contribution made, and validity of the text to follow. If they are pressured with shortage of time and the need to make a decision on the value of the article, or a citation and reference to it in their own work, let this part give answers to all important possible questions, and earn appreciation. Readers and especially followers of the specific research directions will appreciate such a style and will prefer mentioning such work rather than the work of someone else who remained unclear in the introduction and the article was never read due to the lack of time or loss of confidence.

Here are recommended sections, or subsections, or parts of an introduction.

- General overview of the field (basic facts needed to tune the reader to the thesis or paper);

- Problem statement (precise definition and importance);

- Very technical definitions and statements should be avoided (and presented in later text) and instead, good intuition for the involved definitions or facts should be presented and even illustrated if desirable;

- Existing solutions and their criticism (limited normally to only those directly relevant to the contribution of the thesis), a motivation for doing research on the topic should be stated;

- Contributions (proposed solutions; why they are expected to be better; essence of the idea(s) used in proposed solutions);

- Conditions, context, assumptions and limitations of the research done;

- Analysis (theoretical, experimental, simulations, implementations, etc.) done in the thesis; under what conditions and scenarios is the new solution the best one?

- In case of thesis work, it is recommended to add specific statements about the contribution of the thesis author to the thesis work, and the contribution of the thesis to the research field. This is normally done by listing all existing and intended journal and conference publications out of the thesis, which include their authors and titles, and references to proper sections in the thesis.

- The structure and content of the rest of the document is normally outlined at the end of an introduction or Chapter 1 in a single paragraph.

Some sections should normally present the highlights with pointers to later sections and chapters that provide details. The introduction should attempt therefore to present a full version of the article in a concise, readable and intuitively clear form. 


\section{LITERATURE REVIEW: THE NEXT SECTION OR CHAPTER}

Chapter or section 2 should give a full literature review. Many articles present it at the end of the paper, leaving the reader to wonder about the actual contribution until the very end of the text. The literature review (or related work section) should collect all known results relevant to the problem stated, whether or not they are used in the proposed contributions. No additional literature review shall be added in later chapters, where the text could only refer to well known results (e.g. those covered in the undergraduate computer science program such as Dijkstra's shortest path algorithm, sorting algorithms etc.) which can be reasonably assumed to be public knowledge for the particular field. In these cases, they should not be described (that is, if there is a need to describe how a well known shortest path algorithm works, then this should be done in the literature review section).

It is important to underline the need for a clear cut, clear separation line, between existing work and new ideas being presented in the paper. There are in fact three such separation lines: one in each of the abstract, introduction (or Chapter 1) and the most important one between the literature review section and the rest of text. That line should be very sharp.

In some cases, the paper may present minor variations, with major consequences, of an existing solution. In this case, the contribution may look large in the essence, but short in text. This author still advocates to separate these two, even if it means a single paragraph of text in describing novel ideas.

This approach however may not receive positive feedback from reviewers that do not share this philosophy. Separation lines are then needed inside the text itself as a compromise. The main problem with placing separation lines inside the text, rather than by placing content in different sections, is that the separation between existing and new ideas easily remains unclear if the presentation is not very careful. Of course, the assumption here is that the primary intention was to be honest with the contribution made in the article.

One of the major pieces of advice is to do a really thorough literature review on the suggested topic. In most observed cases, however, this does not occur. This is also not really advocated by most supervisors. It is also not emphasized in other papers seen on how to write research articles. We have even seen $P h D$ theses without any literature reviews on the topic. This advice steams from a scientific view of doing research, where the best solution is searched for given model and assumptions. There exist also an innovation view of research, where a good solution is desired for a practical problem. In the scientific point of view, the new solution should be compared with competing solutions, the best existing solutions under particular assumptions, metrics, and models. In the innovation viewpoint, the emphasis is rather on the validation of the new idea, with/out comparing it with something else that exists. A very frequent problem, however, is to mix the two approaches, by attempting to solve a practical problem by using its simple modeling (every model is incorrect but some of them are useful) but evaluating it in a different practical' model (Stojmenović, 2008). Another issue is comparison with solutions that use different metric and assumptions from the one used in new solution (unfair advantage) (Stojmenović, 2008).

While on one hand doing a thorough literature review could prove to be dangerous for the new idea in mind, it is expected to be very rewarding in the long term, as it opens the views to different models, assumptions, different problem statements, and offers material for new contributions with much greater compensation for potentially lost contribution. In fact, ideas are normally credited to original authors anyway, not to those that duplicate it. One of the identified problems is that the existing practice is to merely cite a paper on the subject of study (which happens to be very close to the stated problem and possibly presenting a competing solution), without thoroughly studying it, or describing it properly, which opens the door for an even more dangerous duplication, one that eliminates the excuse of possible independent work.

It is very easy for a reviewer or even examiner to save his time by observing a missing important reference, and claiming that that the particular reference may solve the same problem in a better way. That may or may not be true, but some decisions are not recoverable (e.g. in the case of conference submissions).

The list of references is important for the selection of reviewers, especially when submitting to a journal. Editors normally check the reference list in search of reviewers working on same or similar problem. If the list appears "tiny" on the exact problem statement, editors may search for reviewers on Google Scholar for instance. Reviewers selected 
that way may be subjective in their evaluation if their important relevant work was not cited in the submission ("the paper does not cite me, therefore something is wrong here").

For every discussed reference, it is very important to relate them to the stated problem and contribution in one of several ways: it does not exactly solve the same problem, it solves the same problem but makes different assumptions about the system, it does not meet certain desirable properties (e.g. it is not real-time solution), it has some additional limitations, or it makes the same assumptions but does not work well under certain important conditions and scenarios that are the primary target of the new solution. A clear statement for each identified solution in this respect is recommended. The space allocated to describing existing solutions should also be proportional to its closeness to the new idea and assumptions. Some solutions do not need to be described at all, and a simple convincing statement of why they do not solve the problem at hand may suffice. Other solutions may need a brief description of the general philosophy of the solution before being able to make a similar statement. Otherwise the solution is a candidate to be a competing one, and requires more attention and space. Such existing solutions need clear, concise descriptions of how they work, so that readers can understand a comparison. They are targets for defeat by analytical and/or experimental comparisons. There might be a clear reason why a particular competing solution is inferior to the newly proposed one. Inability to defeat a particular solution certainly leaves a negative impression on readers.

In summary, the literature review should be a critical one, focused around desired outcome and contribution relevant. It should discuss advantages and drawbacks of known solutions that are relevant to the problem studied, and also discuss the relevance of each reviewed item to the topic studied and newly proposed solutions.

\section{THE REMAINING CHAPTERS OR SECTIONS}

The remaining sections/chapters should present new contributions (including conditions, assumptions, and limitations, where appropriate) and their analysis. That is, the very same items listed above should be presented in full, preferably in the same order. Assumptions refer to the simplifications made in the model used, so that the solution can be easily understood, while preserving most properties of a realistic model and enabling easy theoretical and/ or experimental tractability. Analysis could be analytical, by simulation or implementation. Analytical analysis could provide, for example, the proof of validity of the major ideas of the paper. It could lead to a rough estimation of the performance (e.g. message complexity for communication among sensors or average/worst time complexity for computation in sensor processors), calculation of parameter values for simulation, and other relevant properties and findings.

"When presenting an algorithm, first state what the output is and preferably the key idea, before discussing steps" (Ernst, 2005). Pseudo-code description, if used, should include the mnemonic name for the algorithm, its input and output. Such pseudocodes should be preceded in text with clear concise descriptions of same algorithm.

One should always keep in mind that a figure may be worth a thousands words. Important new concepts, and new ideas, should be illustrated by examples and figures as appropriate, to help the reader in understanding them, and to demonstrate one's own understanding of these concepts. This author found most mistakes in student's understandings by simply asking them to give different types of examples. The same is with readers. Examples should not be trivial, but meaningful and helpful.

Figures with examples, and diagrams with performance evaluation, should not be overly repetitive. A new example is welcome if it offers something essentially different and insightful compared to previous ones. Similarly, additional performance diagrams are welcome only if they offer new performance data, substantially different from data in previous diagrams, for the selected set of parameter values. Repetitive diagrams offering similar value for the analysis should be omitted or moved to an appendix of a thesis. The overall contribution is not evaluated by the overall length of a thesis or paper, but by its technical content. In other words, the additional size in page length should be justified by the additional contribution, explanation and insight made.

Captions deserve special attention, which is neglected in a typical written presentation(Milutinović, 1996). Reading only the figure captions of the paper should almost substitute the first rough reading of the entire paper. In case of simulation diagrams, parameter values and protocol names must be clearly 
visible and/or listed in the caption. Captions should include title, description of one or more phenomena that deserve attention, explanation (essential reason for observed behavior) and possibly the implication for the protocol/system design.

It is a very difficult task to find a new solution that is best in all circumstances. The primary task in the simulation part of an article is to identify assumptions, metrics, models and parameter values for which the new solution is better than existing ones. The authors should search for scenarios in which their solution is the best. More details on simulation can be seen in (Stojmenović, 2008). One should not be overly optimistic about new ideas and make unfounded claims. A smaller but justified claim is better than a large unfounded one. Bigger claims open bigger doors for attacks by referees and examiners. Referees may easily turn down the complete idea because of an unsupported large claim, but can also easily accept even a minor contribution if it is well documented and proven.

One of the key pieces of advice is to include all the possible criticisms of your own idea and contribution directly in the article. It is much better that authors criticize their own work and demonstrate good judgment than to leave such pleasure to the examiners and referees. Authors should show that they are in full control of the problem, solutions, and their performance.

\section{ON CONCLUSIONS AND REFERENCES}

Some people read only the abstract and the conclusion. Thus important things missing in the abstract should be placed in the conclusion section. It could state what has been achieved by the current research, and could discuss and reiterate major advantages and drawbacks of the new solution. The most important part of the conclusion section is to list future work that can be done using the results of the current article. This may offer readers with some open problems to study, and such feasible problems could lead to later citations of the article. Sometimes the space can be used to in fact briefly outline some ideas that the author intends to develop further. The ownership of some other possible solutions, not fully explored, to the same or a relevant problem, or subject of your forthcoming different article, can be protected by outlining them briefly in the conclusion section, possibly even with reference to an upcoming article.
One recommendation is to follow a +-+ pattern in the introduction and the main text. That is, to start with positive enthusiastic comments about new work and the contribution, then become realistic and list all the drawbacks and limitations, but then finish on a positive note, with a clear statement about the value of the new contribution. It is important that the reader finishes reading the article with a positive impression. He might be writing a follow up report afterwards.

\section{OVERALL FLOW AND APPEARANCE}

It is important to check if the article has an overall flow, a smooth transition from topic to topic, from familiar information to new information. Within each of the abstract, introduction, or main text, repetitions should be avoided. One statement and its description should be given in a single, most suitable place in that part of the article.

Writing should be clear and concise. "Writing more clearly will help you think more clearly and often reveals flaws or ideas that had previously been invisible even to you" (Ernst, 2005). "Use shorter and more direct phrases wherever possible" (Ernst, 2005). Each concept or algorithm should have a descriptive name. Terms should be normally defined before using them, and should be used precisely and consistently. Ambiguities should be avoided. The text should discuss how related concepts are different and/or similar. Avoid passive voice. Do not use works like "obviously" or "clearly", which my insult reader's intelligence (Ernst, 2005).

Finally, after the scientific presentation is deemed acceptable, it is time to pay more attention to the language used and the overall appearance. It is very important to use proper English grammar and sentence structure, and avoid slangs. Misprints must be corrected. One always expects very professional referees and examiners. Their opinion is partially subjective, compared to, say, an evaluation expected from a knowledgeable robot. A good approach in extracting a positive impression for the subjective part of the overall evaluation is by showing the overall care taken in writing the article.

\section{RELATED WORK ON PRESENTING RESEARCH ARTICLES}

This literature review is limited to items that are not elaborated elsewhere in this article. There is 
plenty of advice for writing research articles that can be found on the Internet. However, most of it deals primarily with language, grammar and formatting issues, and does not go deeply into discussing how to properly and effectively present the essence of the contribution made in a given article.

Instructions for preparing transparencies and oral presentations (Milutinović, 1996) are complete and we found no need to elaborate on them further here. A particular feature in article written by Milutinović (1997) is to use semantics based layout strategy on transparencies.

Alba (2002) presented some brief comments and advice addressing the following problems that students and new researchers face: structure the document, formatting guides, content, readability, electronic edition and diffusion. The suggested structure of the document is: Introduction, Problems (this part includes literature review), Resolution methods, Experiments, Results, Conclusions, and References. Resolution methods should stress the novelty of the method and approach, a specific non-ambiguous explanation of the method (e.g. pseudocodes), mathematical or formal issues of proposed techniques, parameters and most important decisions made to select these methods or techniques, plans to solve the problems with proposed appropriate methods, and expected results after having done so. The experiments section should present the goals and sets of experiments, parameters, algorithms and problem instances intended for use (preferably in table form), measures, statistical analysis, and criteria to judge the value of the results, steps to follow to get the results with justification.

Woodford (1999) lists the overall steps in writing a journal article, dissertation or grant proposal, in a brief note, while the full text is available in his book. It begins from asking whether the time is right for writing, to analyzing and possibly answering the examiner's remarks. Ernst (2005) discusses rejection: "In most cases, reviews offer an opportunity to improve the work". To avoid reputation of submitting "a half-baked work", only submit to obtain new information (including concerns not predicted in advance).

Recommendations for writing articles also depend on the research field. Sherrill (2003) briefly described recommendations for writing articles in the chemistry discipline. The parts of a paper are: $\mathrm{Ab}$ stract, Introduction, Methods (or Theoretical Methods), Results and Discussion, and Conclusions. Simi- lar structure for papers can be found in other fields, e.g. psychology.

The structure of a research paper is less rigorous in mathematics field. Normally papers are collections of theorems and proofs, and every known proof from other sources is cited in the text where needed. Here are a few of pieces of advice by Cheney (2000) that are found to be quite relevant for computing and engineering articles (e.g. description of algorithms, protocols and systems). "Mathematics is preeminent in its striving for absolute precision in its formal written text. Precision in writing is not easily attained, but one always begins by using the correct word at the proper place and by carefully constructing each sentence. We also advise against the use of slang, colloquialisms, and other nonstandard linguistic devices" (Cheney, 2000).

"Use English descriptions and English text in preference to mathematical symbolism wherever possible." (Cheney, 2000). "It makes for smoother reading. Mathematical symbolism is by its nature intimidating, even to mathematicians. There is nothing so daunting as having to read a page of formulas! Keep the formulas to a minimum and avoid symbols if ordinary language will do as well. There may be cases where, for good reason, one wishes to violate this rule." (Cheney, 2000).

Another good reason to avoid math formalism is the impact of possible misprints. A single misprint anywhere in a fully mathematical formula and the expression can have disastrous consequence for the interpretation and understanding, not only of that particular formula but the rest of the text. In some cases, the reader is even unable to continue reading the article. On the other hand, a single misprint in an English sentence, even an awkward English sentence, allows the reader to automatically correct and continue reading. In some cases, the best approach is to give a math expression followed by its decoding with analogous statements in English. Sometimes it is simply not easy to avoid math symbolism without loosing precision.

\section{WRITING GOOD SYSTEMS PAPERS}

The advice so far was applicable to technical papers in general. To address the readership of IEEE Transactions on Parallel and Distributed Systems, we address some specific problems in "systems" papers, following closely (Levin and Redell, 1983). They listed thirty-odd questions to help writing a 
better technical paper. Questions related to originality of ideas, focus, presentation and writing style are close to the discussion here, often given from a complementary perspective. Questions specific to systems papers are about reality, lessons learned and choices made. Does the paper describe something that has actually been implemented? If so, how has it been used, and what has this usage shown about the practical importance of the ideas? Otherwise, do the ideas justify publication now? What authors learned and what should the reader learn from the paper? How generally applicable are these lessons? What were the alternatives considered at various points, why the choices were made the way they were, and did the choices turn out to be right? How realistic are assumptions? Does the formal model, if presented, give new information, and is it supported by any deep theorem?

According to Levin and Redell (1983), writing a good paper is a hard work, but you will be rewarded by a broader distribution and greater understanding of your ideas within the community of journal and proceedings readers.

\section{REFERENCES}

Alba, E. (2002) Writing a scientific/technical report. [online] Available from: www.lcc.uma.es/ eat/pdf/sw.pdf [acessed 27 June 2011].

Cheney, W. (2000) Advice for students writing reports, theses, and dissertations. Department of Mathematics, University of Texas at Austin. [online] Available from: www.ma.utexas.edu/users/cheney/ [acessed 21 August 2011].
Ernst, M. (2005) Writing a technical paper. [online] Available from: www.cs.washington.edu/homes/mernst/ advice/write-technical-paper.html [acessed 19 April 2011].

Kalosha, H., Nayak, A., Ruehrup, S., Stojmenović, I. (2008) Select and protest based beaconless georouting with guaranteed delivery in wireless sensor networks. IEEE INFOCOM, 13-18 April 2008 Arizona.

Levin, R., Redell, D. (1983) How (and how not) to write a good systems paper. ACM Operating Systems Review. 17 (3), 35-40.

Milutinović, V. (1996) The best method for presentation of research results. IEEE TCCA Newsletter [online] Available from: http://tab.computer.org/tcca/news/ sept96/bestmeth.pdf [acessed 03 August 2011].

Milutinović, V. (1997) A good method to prepare and use transparencies for research presentations. IEEE TCCA Newsletter. [online] Available from: http://tab.computer.org/tcca/news/sept96/bestmeth.pdf [acessed 16 June 2011].

Sherrill, D. (2003) Writing journal articles. Georgia Institute of Technology. [online] Available from: vergil. chemistry.gatech.edu/resources/writing-papers.pdf [acessed 13 May 2011].

Stojmenović, I. (2000) Recursive algorithms in computer science courses. IEEE Transactions on Education. 43 (3), 273-276.

Stojmenović, I. (2008) Simulations in wireless sensor and ad hoc networks. IEEE Communications Magazine. 46 (12), 102-107.

Woodford, P. (1999) Writing a journal article, dissertation, or research-grant proposal: Steps to for graduate students and others. CBE Views. 22 (1), 12-13.

\section{PREZENTOVANJE REZULTATA U OKVIRU ORIGINALNOG NAUČNOG RADA IZ OBLASTI RAČUNARSKIH I TEHNIČKIH NAUKA}

\section{Rezime:}

Ovaj članak daje opšte smernice u pisanju istraživačkih članaka u oblastima koje su vezane za računarstvo, informacione tehnologije i tehničke nauke. $U$ uspešnom članku je najvažnije da se ponovi opis glavnog doprinosa četiri puta: u naslovu, apstraktu, uvodu (ili prvom poglavlju) i u tekstu. To znači da se napišu čitke i što potpunije verzije rada upotrebom 10, 100, 1.000 i 10.000 reči. To odgovara i smanjenju broja čitalaca pomenutih delova članaka. Koliko god je to moguće, svaki od ovih delova treba da sadrži, ovim redom: formulisanje problema, postojeća rešenja, novo rešenje (rešenja), pretpostavke i ograničenja, analizu, simulaciju i poređenje sa najboljim konkurentskim rešenjima.

\section{Ključne reči:}

informatika, originalni naučni rad, apstrakt, naslov.

Received: September 15th, 2011

Correction: October 22nd, 2011

Accepted: November 15th, 2011 